\title{
Biomarkers in fibromyalgia: a review
}

This article was published in the following Dove Press journal:

Current Biomarker Findings

21 February 2014

Number of times this article has been viewed

\section{Camillo Giacomelli* \\ Francesca Sernissi* \\ Alessandra Rossi \\ Stefano Bombardieri \\ Laura Bazzichi}

Rheumatology Unit, Department of Clinical and Experimental Medicine, University of Pisa, Pisa, Italy

*These authors contributed equally to the manuscript
Correspondence: Camillo Giacomelli Rheumatology Unit, Department of Clinical and Experimental Medicine, University of Pisa, Via Roma,

67, 56126 Pisa, Italy

Email camillo.giacomelli@for.unipi.it
Abstract: Fibromyalgia is a common syndrome diagnosed by clinical criteria. The main symptom of fibromyalgia is pain, but patients frequently also complain about other nonspecific symptoms, such as headache, sleep disturbance, mood disorder, and cognitive impairment. In the light of the multifactorial origin of the disease and of the lack of objective diagnostic findings, several attempts have been made to find a reliable biomarker. For this reason, over the years, a number of patients and various biological samples have been studied, using many different approaches and techniques. Despite this, none of these studies has been able to find the proper biomarker. The aim of this review is to provide a critical overview of the current environment characterizing the search for fibromyalgia biomarkers.

Keywords: genetics, proteomics, oxidative stress, fibromyalgia

\section{Introduction}

Fibromyalgia (FM) is a common syndrome that affects mainly women, with a worldwide prevalence of $2.7 \%$ and a male:female ratio of $1: 3{ }^{1}$ According to the recent American College of Rheumatology criteria, ${ }^{2,3}$ FM is a nonautoimmune rheumatic disease characterized by widespread pain and a plethora of nonspecific symptoms, such as cognitive impairment, headache, sleep disturbance, and mood disorder. These criteria focus on pain and related symptoms, and overlap with the first classification criteria ${ }^{4,5}$ (1990), which defined FM as a condition characterized by pain and the presence of multiple tender points. Irrespective of the classification criteria applied, the diagnosis of FM remains basically clinical, and despite the fervent research for FM biomarkers, none of the suggested candidates has yet proven to be convenient, reliable, specific, and sensitive. In fact, in the absence of any classical physiologic alteration, the diagnosis of FM remains clinical, with patients' reported symptoms (tenderness and pain) used as the gold standard for the diagnosis. Starting from these assumptions, there is a real risk of misdiagnosis, with relevant socioeconomic and treatment consequences. ${ }^{6}$

However, researchers have tried to find a biomarker for this syndrome, ${ }^{7}$ for both diagnostic and therapeutical purpose.

\section{Genetics}

Since it is known that FM syndrome often occurs in more than one member of a family, ${ }^{8}$ it was reasonable to predict that genetic predisposition or other biochemical dysfunctions may be important to the development and/or perpetuation of FM. 
Genetic factors are known to influence predisposition to FM, but no specific genes have been definitively involved. ${ }^{8}$

Many authors have discussed the implication of genetic alterations in FM. In particular, they have focused attention on the alteration of genes involved in nociception, such as the monoamine system, substance $\mathrm{P}$ receptors, dopamine transporters, and alpha 1 antitrypsin. The genetic alterations found in FM are showed in Table 1.

\section{Hematological alterations Autoantibodies}

Research on hematologic alterations in FM is desirable. Many studies have been conducted in an effort to find alterations in hematological findings, such as autoantibody levels.

With respect to autoimmunity, one of the investigated antibodies was antipolymer antibody (APA), found in the sera of women with silicone breast implants who presented FM-like symptoms. Wilson et al evaluated APA levels in FM patients and found a higher percentage of APA positivity in the FM group (67\%). ${ }^{16}$ However, these results were not confirmed in other studies. ${ }^{17} \mathrm{~A}$ later study, involving a cohort of 285 FM Italian patients and 100 healthy controls, evaluated APA levels and their correlation with disease severity and cytokine levels. ${ }^{18}$ APA-positivity was detected in only $21.05 \%$ of FM patients and $15 \%$ of healthy controls, with no significant difference between the two groups. Despite the previous study findings, APA cannot be considered as a FM diagnostic biomarker. However, the presence of APA might be useful in identifying a subgroup of FM patients with more severe symptoms; in fact, in one study, slightly higher APA levels were found in patients with severe disease, and a positive correlation between APA levels and scores on the Fibromyalgia Impact Questionnaire (FIQ) was reported. ${ }^{18}$

A few other antibodies were also studied in FM. In one study, the levels of antibodies to 5-hydroxytryptamine, gangliosides, and phospholipids were found to be higher in patients with FM and chronic fatigue syndrome (CFS). ${ }^{19}$ In this work the authors reported a statistically significant difference between FM and CFS in terms of serotonin, ganglioside, and phospholipid antibodies. In addition, $49 \%$ of FM and $17 \%$ of the CFS patients had all three antibodies, and $70 \%$ and $55 \%$ of patients, respectively had at least two of these antibody types. Based on these finding, the authors proposed the FM syndrome definition of "psychoneuroendocrinological autoimmune diseases", aimed at emphasizing the etiology of FM.

Moreover, Nishikai et al have proposed anti-68/48 kDa and the anti-45 $\mathrm{kDa}$ as possible markers for FM and CFS, specially describing a specific disease subset, which is characterized by a secondary FM form and the copresence

Table I Genetic alteration in FM

\begin{tabular}{|c|c|c|c|}
\hline Authors & Year & Reference & Findings \\
\hline Caccamo et al & 2013 & 9 & $\begin{array}{l}\text { The authors compared the frequency of gene polymorphisms of selected CYP P450 metabolizing } \\
\text { enzymes and the frequency of the xenobiotic AHR, in three cohorts and } 80 \text { FM patients. They found } \\
\text { a significantly higher frequency of CYP and AHR polymorphisms in patients compared with controls }\end{array}$ \\
\hline Smith et al & 2012 & 10 & $\begin{array}{l}\text { The authors studied } 350 \text { genes involved in nociception, inflammation, and mood disorders in a large } \\
\text { (496) cohort of FM patients and } 346 \text { pain-free controls } \\
\text { They found significant differences in three genes involved in amine transport: GABRB3, TAARI, and GBPI }\end{array}$ \\
\hline Xiao et al & 2012 & 8 & $\begin{array}{l}\text { The authors evaluated the Val66Met polymorphism (SNP) in the BDNF gene, in } 95 \mathrm{FM} \text { patients. } \\
\text { The frequency of this polymorphism did not differ from healthy controls, but the subgroup with } \\
\text { BDNF Val66Val showed higher CRP and BMI }\end{array}$ \\
\hline Potvin et al & 2010 & 11 & $\begin{array}{l}\text { The authors compared } 58 \mathrm{FM} \text { patients with healthy controls to evaluate thermal pain thresholds and } \\
\text { the correlation with the 5-HTTLPR polymorphism. They found higher pain thresholds in the healthy } \\
\text { controls compared with those in the FM patients and no correlation with 5-HTTLPR }\end{array}$ \\
\hline Cohen et al & 2009 & 12 & $\begin{array}{l}\text { The authors studied } 209 \mathrm{FM} \text { patients for the Val/Met polymorphism in the COMT gene. They } \\
\text { observed an association between FM and the COMT Val(I58)Met polymorphism }\end{array}$ \\
\hline Ablin et al & 2009 & 13 & $\begin{array}{l}\text { The authors studied the frequency of the polymorphism of three candidate genes in } 87 \text { FM patients: } \\
\text { the substance } P \text { receptor }(T A C R I) \text { I } 354 \mathrm{G}>\mathrm{C}, D A T \text {, and AAT. They didn't find differences among the } \\
\text { FM patients }\end{array}$ \\
\hline Tander et al & 2008 & 14 & $\begin{array}{l}\text { The authors did not find any difference in the COMT ( } r s 4680) \text { and 5-HT2A ( } r s 63 \text { I3 and rs63II) } \\
\text { genes in } 80 \mathrm{FM} \text { patients }\end{array}$ \\
\hline Gürsoy et al & 2008 & 15 & $\begin{array}{l}\text { The authors evaluated the MAO-A and MAO-B polymorphism in } 107 \text { FM patients. They suggested } \\
\text { the possible relation between the high-activated MAO-A allele } 3 \text { in the occurrence of FM }\end{array}$ \\
\hline
\end{tabular}

Abbreviations: 5-HT2A, 5-hydroxytryptamine (serotonin) receptor 2A; 5-HTTLPR, serotonin-transporter-linked polymorphic region; AHR, sensor aryl hydrocarbon receptor; BDNF, brain-derived neurotrophic factor; CYP, cytochrome; BMI, body mass index; COMT, catechol-O-methyltransferase; CRP, C-reactive protein; DAT, dopamine transporter; FM, fibromyalgia; GABRB3, gamma-aminobutyric acid (GABA) A receptor, beta 3; GBPI, interferon-induced guanylate-binding protein I; MAO, monoamine oxidase; Met, methionine; SNP, single nucleotide polymorphism; TAARI, trace amine-associated receptor I; TACRI, tachykinin receptor I; Val, valine. 
of psychiatric disorders. ${ }^{20}$ They found that anti $68 / 48 \mathrm{kDa}$ protein was present in $15.6 \%$ of FM patients and also, that the antibody against $45 \mathrm{kDa}$ protein was present in $37.1 \%$ of patients with secondary FM. They also showed that the presence of $68 / 48 \mathrm{kDa}$ was most frequent in patients with hypersomnia and cognitive impairment. For this reason, these antibodies were not able to support a FM diagnosis; however, they may be useful as markers for a particular subset of FM.

Many patients with FM or other rheumatic conditions, such as rheumatoid arthritis or Sjögren's syndrome, present with an alteration of thyroid function and have been frequently diagnosed with a thyroid autoimmune disease. In a large cohort of 120 FM patients, Bazzichi et al evaluated the presence of thyroid abnormalities and autoimmunity, ${ }^{21}$ observing that FM patients had normal thyroid hormone values but that $41 \%$ of the patients had at least one thyroid antibody. The presence of autoimmune thyroiditis was also associated with the typical FM symptoms.

In a later study, Bazzichi et al ${ }^{22}$ also observed that the presence of thyroid disease worsened FM symptoms. They evaluated FM comorbidity in patients with Hashimoto's thyroiditis (HT) with or without subclinical hypothyroidism (SCH), and in patients with $\mathrm{SCH}$ alone; as well as the impact of antithyroid autoimmunity and SCH on FM comorbidity. A total of 52 patients, 39 affected by HT with or without $\mathrm{SCH}$ and 13 with SCH alone were compared with $37 \mathrm{FM}$ patients and 25 healthy subjects. The researchers found that $31 \%$ of HT subjects and none of the SCH patients had FM as a comorbidity. Further, $33.3 \%$ of HT patients without SCH and $28.5 \%$ of HT patients with SCH had FM comorbidity. Moreover, they observed that the presence of thyroid autoantibodies seemed to worsen the symptoms of FM. The patients with both HT and FM, when compared with patients with $\mathrm{SCH}$, had a significantly higher incidence of positive tender points (100\% versus [vs] $23 \%$ ), diffuse pain ( $92 \%$ vs $8 \%$ ), fatigue ( $92 \%$ vs $54 \%$ ), paresthesias ( $83 \%$ vs $31 \%$ ), nonrestful sleep ( $75 \%$ vs $31 \%$ ), and affective disorder ( $58 \%$ vs $23 \%$ ), higher mean FIQ scores (indicating greater impact) $(58.70 \pm 21.35$ vs $17.39 \pm 14.48)$, higher mean visual analog scale (VAS) pain scores $(7.50 \pm 2.58$ vs $1.54 \pm 2.54)$, and higher mean VAS fatigue scores $(5.67 \pm 2.42$ vs $0.38 \pm 0.77$ ). They thus supposed a possible relationship between FM and thyroid autoimmunity, suggesting that the presence of thyroid autoimmunity could play a pivotal role in the pathogenesis of FM and that it could help identify patients with a worse prognosis.

Thyroid implication in the etiopathogenesis of FM was also confirmed by other authors who studied the role of thyroperoxidase antibody (TPO Ab). Repeated studies have demonstrated a high prevalence of thyroid autoantibodies in FM patients, but nevertheless, the role of TPO $\mathrm{Ab}$ in FM remains unclear. In 2012, Suk et al ${ }^{23}$ explored the prevalence of TPO Ab in FM patients with normal thyroid function and studied the potential correlation between autoimmunity and FM clinical profile. They confirmed the observation of a higher frequency of TPO Ab in FM. However, typical findings of FM were not influenced by the presence or absence of TPO Ab. These results confirm that autoimmune thyroiditis may have a role in the development of FM but also, that the presence of TPO Ab itself may not be correlated with the variety and severity of FM symptoms. Similar results were previously reported by Ribeiro and Proietti, who suggested the correlation between FM and thyroid autoimmunity. ${ }^{24}$

Unfortunately, the above mentioned antibodies have no appreciable characteristics of sensitivity and specificity, and moreover, they need to be validated before being considered strong and useful diagnostic biomarkers for FM.

\section{Oxidative stress}

Several articles reported a linkage between the alteration of oxidative stress response and FM. In Table 2, we report the main articles published in this field. A putative involvement of oxidative stress and FM stems from the observation of clinical response to antioxidative drugs, such as coenzyme Q10 and others vitamins. For instance, few works describe the clinically significant improvement of FM patients treated with oral supplementation of coenzyme Q10, a cofactor known to play an important role in the scavenging of reactive oxygen species. ${ }^{25,26}$ Coenzyme Q10 is a membrane stabilizer and a vital cofactor in the mitochondrial electron transport chain, enabling the generation of adenosine triphosphate (ATP). Cordero et al have published work on the efficacy of coenzyme Q10 in relieving pain and other FM symptoms. ${ }^{25,26}$ Similar results were found by Miyamae et al, who studied the oral supplementation with coenzyme Q10 in ten juvenile FM patients. ${ }^{27}$ However, even if oxidative stress plays a pivotal role in FM, there aren't any specific biomarkers able to evaluate it.

\section{Cytokines}

Although FM is not an inflammatory disease, many authors have reported the alteration of several cytokines in FM patients. These findings suggest a possible subthreshold inflammation. In a recent meta-analysis, the authors reviewed the literature that investigated cytokines in FM patients. ${ }^{34}$ The results, 
Table 2 Alteration of oxidative response system and FM

\begin{tabular}{|c|c|c|c|}
\hline Authors & Year & Reference & Findings \\
\hline Meeus et al & 2013 & 28 & The authors hypothesized that the increase of ROS in FM was due to mitochondria dysfunction \\
\hline Castro- & 2013 & 29 & The authors investigated the possible association between mitochondrial biogenesis and oxidative stress in \\
\hline Marrero et al & & & $\begin{array}{l}23 \text { patients with CFS and in } 20 \text { with FM. They found decreased levels of coenzyme QI0 and ATP, together } \\
\text { with increased levels of lipid peroxidation. These results suggest a mitochondria involvement in FM }\end{array}$ \\
\hline Neyal et al & 2013 & 30 & $\begin{array}{l}\text { The researchers studied plasma nitrite levels, total antioxidant status, total oxidant status, and oxidative } \\
\text { stress index in } 33 \mathrm{FM} \text { patients and } 3 \mathrm{I} \text { healthy controls. The main result was the higher level of total } \\
\text { oxidant status and oxidative stress index in FM patients with respect to controls }\end{array}$ \\
\hline Bote et al & 2012 & 31 & $\begin{array}{l}\text { The authors studied circulating "neuroendocrine-stress" biomarkers (CRH, ACTH, cortisol, NA, eHsp72, } \\
\text { serotonin, and IGF-I). Serum cytokines levels and CRP concentrations were also determined. FM patients } \\
\text { showed an inflammatory state accompanied by an altered stress response. This was mainly manifested by } \\
\text { high circulating levels of IL-8 and high levels of stress molecules }\end{array}$ \\
\hline $\begin{array}{l}\text { Giacomelli } \\
\text { et al }\end{array}$ & 2011 & 32 & $\begin{array}{l}\text { By a proteomic approach, we found some altered proteins in saliva of FM patients. In particular, regarding } \\
\text { oxidative stress, we found higher levels of calgranulin A and C, which are involved in oxidative stress response }\end{array}$ \\
\hline Altindag et al & 2007 & 33 & $\begin{array}{l}\text { The authors studied paraoxonase and arylesterase levels in } 42 \mathrm{FM} \text {, and they found a decrease of } \\
\text { antioxidative status in FM patients }\end{array}$ \\
\hline
\end{tabular}

Abbreviations: ACTH, adrenocorticotropic hormone; ATP, adenosine triphosphate; CFS, chronic fatigue syndrome; $\mathrm{CRH}$, corticotropin-releasing hormone; CRP, C-reactive protein; eHsp, extracellular heat shock protein; FM, fibromyalgia; IGF, insulin-like growth factor; IL, interleukin; NA, noradarenaline; ROS, reactive oxygen species.

extrapolated from 1,255 FM patients and 800 healthy subjects, emphasized the role of cytokines, especially for interleukin 6 and interleukin 8 . However, the role of cytokines and FM still remains unclear. In Table 3, we summarized the principal findings of studies investigating cytokine levels and FM.

\section{Other hematological findings}

Over the last 10 years, the literature has reported several other attempts to find reliable biomarkers for FM. For instance, two independent authors found higher serum hyaluronic acid levels in FM patients compared with healthy controls. However, this data has not yet been confirmed, and the significance of this alteration still remains unclear. ${ }^{41,42}$

Another field of interest is the evaluation of amino acids in the plasma of FM patients. Maes et al found significant lower levels of branched-chain amino acids in the plasma of FM patients, and they speculated that the alteration of branched-chain amino acids could lead to a lack in the supply of energy to the muscle and to altered protein synthesis in the muscles. ${ }^{43}$ In addition, more recently, an Italian research group found the alteration of several amino acid levels in FM patients. ${ }^{44}$ In fact, they evaluated the levels of 20 amino acids in plasma from 34 FM patients and found a significantly lower level of taurine, alanine, tyrosine, valine, methionine, phenylalanine, and threonine; these findings could indicate a possible bowel malabsorption of the mentioned amino acids. ${ }^{44}$

Because of the considerable use of antidepressant drugs, especially serotonin-noradrenalin reuptake inhibitors and selective serotonin reuptake inhibitors, serotonin levels in FM patients cannot be considered as a biomarker. In fact,

Table 3 Cytokines and FM

\begin{tabular}{|c|c|c|c|}
\hline Authors & Year & Reference & Findings \\
\hline Bote et al & 2013 & 35 & $\begin{array}{l}\text { The authors evaluated the effect of moderate physical activity on inflammatory serum components, such as } \\
\text { IL-8, chemotaxis activity and } \mathrm{O}_{2}(-) \text { production by neutrophils, IL-I } \beta \text {, TNF- } \alpha \text {, IL-6, IL-I0, and IL-I8 release } \\
\text { by monocytes, and stress (cortisol, NA, and eHsp72). The authors found that a single session of moderate } \\
\text { exercise led to an improvement of the inflammatory status and a decrease in stress molecules }\end{array}$ \\
\hline $\begin{array}{l}\text { Pernambuco } \\
\text { et al }\end{array}$ & 2013 & 36 & $\begin{array}{l}\text { The authors evaluated plasma levels of IL-I7 in } 58 \text { FM patients. They found increased levels of IL-I7 and a } \\
\text { positive correlation between IL- I } 7 \text { levels and other cytokines (IL-2, IL-4, IL- I0, and TNF and IFN } \gamma \text { ) }\end{array}$ \\
\hline Ross et al & 2010 & 37 & $\begin{array}{l}\text { The authors evaluated various cytokines and their relationship between } \mathrm{GH} \text { response and physical exercise. } \\
\text { They found higher values of IL- } 6 \text { and IL- } 8 \text { in general and higher levels of GH in response to exercise, in FM }\end{array}$ \\
\hline $\begin{array}{l}\text { lannuccelli } \\
\text { et al }\end{array}$ & 2010 & 38 & $\begin{array}{l}\text { They found higher levels of IL-IRA, IL-6, IL-I0, and TNF- } \alpha \text { in FM patients compared with tension-type } \\
\text { headache patients and healthy controls }\end{array}$ \\
\hline Togo et al & 2009 & 39 & $\begin{array}{l}\text { They studied the cytokine levels fluctuation over time in eight FM patients compared with the healthy } \\
\text { subjects. FM patients showed a trend to increased IL- I } 0 \text { levels during the night compared with controls }\end{array}$ \\
\hline $\begin{array}{l}\text { Bazzichi } \\
\text { et al }\end{array}$ & 2007 & 40 & $\begin{array}{l}\text { They studied } 80 \text { FM patients compared with } 45 \text { healthy subjects, finding higher levels of IL- I0, IL-8, and } \\
\text { TNF- } \alpha \text { in the FM patients compared with the control group }\end{array}$ \\
\hline
\end{tabular}

Abbreviations: eHsp, extracellular heat shock protein; FM, fibromyalgia; $\mathrm{GH}$, growth hormone; IFN, interferon; IL, interleukin; NA, noradrenaline; $\mathrm{O}_{2}(-)$, reactive oxigen species; TNF, tumor necrosis factor. 
one study reported low serum levels of 5-hydroxytryptamine (5-HT) in FM sera with respect to healthy controls and rheumatoid arthritis patients but no differences in serum 5-HT between FM and patients affected by different psychiatric conditions. ${ }^{45}$ However, there is also evidence suggesting that FM patients may have alterations - both genetic ${ }^{46}$ and functional $\mathrm{l}^{47}$ - in the expression of the 5-HT transporters. Another study found alterations of 5-HT, somatomedin C (a peptide-related to the calcitonin gene), calcitonin, and cholecystokinin, and possible indicators of widespread FM pain ${ }^{48}$ however, these results came from single observations, and therefore validation is needed before conclusions could be drawn. In a recent work published by Ablin et al, leptin levels were studied..$^{49}$ The authors don't observed differences in leptin levels in FM patients compared with the controls.

\section{Proteomics}

In the last few years, proteomic research has been carried out with the main goal of biomarker discovery. In the wake of the interesting results previously reported in rheumatic conditions, such as rheumatoid arthritis, Sjögren's syndrome, and systemic sclerosis, which are known to frequently overlap with FM syndrome, ${ }^{50-52}$ an Italian research group also investigated the salivary profile of FM patients. ${ }^{53}$ From the analysis of whole saliva from $22 \mathrm{FM}$ patients, the most interesting result was the observation of a significant overexpression of transaldolase (sensitivity of $77.3 \%$ and specificity of $84.6 \%$ ) and phosphoglycerate mutase 1 (PGM-1) in FM patients with respect to healthy controls. Transaldolase is an enzymatic protein involved in the oxidative phase of the pentose phosphate pathway. ${ }^{54}$ This enzyme could be overexpressed due to an attempt to limit oxidative damage in tissues; in fact, transaldolase is the rate-limiting enzyme of the nonoxidative part of the pentose phosphate pathway, and as such, it plays a pivotal role in the regulation of the synthesis of nicotinamide adenine dinucleotide phosphate-oxidase (NADPH), as a reductive equivalent.

In addition, researchers found a different expression of several proteins: calgranulin A, calgranulin C, cyclophilin A, profilin 1, Rho guanosine diphosphate (GDP)-dissociation inhibitor 2, proteasome subunit-a-type-2, and haptoglobinrelated protein precursor. ${ }^{53}$ These proteins have an important role in the immune response, in the cytoskeleton remodeling, and in the inflammatory process, but their role in FM still remains controversial.

\section{Conclusion}

Despite the number of alterations reported in the literature, the diagnosis of FM remains mainly clinical. In fact, the alterations found in FM are aspecific and do not have a high grade of sensitivity and specificity. Considering its multifaceted nature and the plethora of symptoms that characterized this syndrome, it is difficult to think that FM can be explained or diagnosed on the basis of a single biomarker.

For example, thyroid involvement is known to worsen various symptoms in FM patients, especially pain, fatigue, and nonrestful sleep. ${ }^{21}$ Also APAs were found to be higher in patients with a severe condition (indicated by increased FIQ scores). ${ }^{18}$ Another example of how biomarkers could be used to better assess both syndrome severity and therapy response is represented by plasma coenzyme Q10 levels: it has been shown that where supplementation with coenzyme Q10 obviously led to increased plasmatic levels, at the same time, patients seemed to report an improvement of pain. ${ }^{25,26}$

However, the results observed in the literature are not consistent and far from allow the use of such biomarkers, in clinical practice, to assess patients' outcomes in an effective and reliable way. Nonetheless, the genetic studies discussed here, despite being nonhomogeneous in terms of study design and methods, were focused on the same pathway (nociception) and found alterations in biogenic amine expression (5HT, noradrenaline, and dopamine) and metabolism (catechol-O-methyltransferase [COMT] and monoamine oxidase [MAO] enzymes). These alterations, indeed, support the rational on which the more effective FM pharmacological treatments are based: selective serotonin reuptake inhibitors and serotonin-noradrenalin reuptake inhibitors.

Some authors discredit even the existence of FM. Despite this, the observed alterations indicate that large clinical trials, with large cohorts of homogeneous patients stratified according to the different subsets of disease, would be able to identify and validate clusters of biomarkers that could be useful for the assessment and monitoring of specific FM symptoms and disorders (eg, widespread pain, fatigue, mood and sleep disorders, gastrointestinal disorders, etc). From the various papers cited in this review, the main physiologic alterations involved in FM are those related to inflammatory response (cytokines), stress oxidative response, and thyroid function. However, although the molecules found to be implicated in the etiopathogenesis of FM are not constitutive biomarkers, they could play an important role in specific subsets of the disease; if these putative biomarkers were to be successfully validated and meet specific requirements of feasibility and reproducibility, they could eventually be useful for diagnostic and therapeutic purposes.

\section{Disclosure}

The authors report no conflicts of interest in this work. 


\section{References}

1. Queiroz LP. Worldwide epidemiology of fibromyalgia. Curr Pain Headache Rep. 2013;17(8):356.

2. Wolfe F, Clauw DJ, Fitzcharles MA, et al. The American College of Rheumatology preliminary diagnostic criteria for fibromyalgia and measurement of symptom severity. Arthritis Care Res (Hoboken). 2010;62(5):600-610.

3. Wolfe F, Clauw DJ, Fitzcharles MA, et al. Fibromyalgia criteria and severity scales for clinical and epidemiological studies: a modification of the ACR Preliminary Diagnostic Criteria for Fibromyalgia. J Rheumatol. 2011;38(6):1113-1122.

4. Salaffi F, Sarzi-Puttini P. Old and new criteria for the classification and diagnosis of fibromyalgia: comparison and evaluation. Clin Exp Rheumatol. 2012;30(6 Suppl 74):S3-S9.

5. Wolfe F, Smythe HA, Yunus MB, et al. The American College of Rheumatology 1990 criteria for the classification of fibromyalgia. Report of the Multicenter Criteria Committee. Arthritis Rheum. 1990;33(2):160-172.

6. Di Franco M, Iannuccelli C, Bazzichi L, et al. Misdiagnosis in fibromyalgia: a multicentre study. Clin Exp Rheumatol. 2011; 29(6 Suppl 69):S104-S108.

7. Bazzichi L, Rossi A, Giacomelli C, Bombardieri S. Exploring the abyss of fibromyalgia biomarkers. Clin Exp Rheumatol. 2010;28(6 Suppl 63): S125-S130.

8. Xiao Y, Russell IJ, Liu YG. A brain-derived neurotrophic factor polymorphism Val66Met identifies fibromyalgia syndrome subgroup with higher body mass index and C-reactive protein. Rheumatol Int. 2012;32(8):2479-2485.

9. Caccamo D, Cesareo E, Mariani S, et al. Xenobiotic sensor- and metabolism-related gene variants in environmental sensitivity-related illnesses: a survey on the Italian population. Oxid Med Cell Longev. 2013;2013:831969.

10. Smith SB, Maixner DW, Fillingim RB, et al. Large candidate gene association study reveals genetic risk factors and therapeutic targets for fibromyalgia. Arthritis Rheum. 2012;64(2):584-593.

11. Potvin S, Larouche A, Normand E, et al. No relationship between the ins del polymorphism of the serotonin transporter promoter and pain perception in fibromyalgia patients and healthy controls. Eur J Pain. 2010;14(7):742-746.

12. Cohen H, Neumann L, Glazer Y, Ebstein RP, Buskila D. The relationship between a common catechol-O-methyltransferase (COMT) polymorphism val(158) met and fibromyalgia. Clin Exp Rheumatol. 2009;27(5 Suppl 56):S51-S56.

13. Ablin JN, Bar-Shira A, Yaron M, Orr-Urtreger A. Candidate-gene approach in fibromyalgia syndrome: association analysis of the genes encoding substance $\mathrm{P}$ receptor, dopamine transporter and alpha1antitrypsin. Clin Exp Rheumatol. 2009;27(5 Suppl 56):S33-S38.

14. Tander B, Gunes S, Boke O, et al. Polymorphisms of the serotonin-2A receptor and catechol-O-methyltransferase genes: a study on fibromyalgia susceptibility. Rheumatol Int. 2008;28(7):685-691.

15. Gürsoy S, Erdal E, Sezgin M, et al. Which genotype of MAO gene that the patients have are likely to be most susceptible to the symptoms of fibromyalgia? Rheumatol Int. 2008;28(4):307-311.

16. Wilson RB, Gluck OS, Tesser JR, Rice JC, Meyer A, Bridges AJ. Antipolymer antibody reactivity in a subset of patients with fibromyalgia correlates with severity. J Rheumatol. 1999;26(2):402-407.

17. Wolfe F. "Silicone related symptoms" are common in patients with fibromyalgia: no evidence for a new disease. J Rheumatol. 1999;26(5): 1172-1175.

18. Bazzichi L, Giacomelli C, De Feo F, et al. Antipolymer antibody in Italian fibromyalgic patients. Arthritis Res Ther. 2007;9(5):R86.

19. Klein R, Berg PA. High incidence of antibodies to 5-hydroxytryptamine, gangliosides and phospholipids in patients with chronic fatigue and fibromyalgia syndrome and their relatives: evidence for a clinical entity of both disorders. Eur J Med Res. 1995;1(1):21-26.
20. Nishikai M, Tomomatsu S, Hankins RW, et al. Autoantibodies to a 68/48 $\mathrm{kDa}$ protein in chronic fatigue syndrome and primary fibromyalgia: a possible marker for hypersomnia and cognitive disorders. Rheumatology. 2001;40(7):806-810.

21. Bazzichi L, Rossi A, Giuliano T, et al. Association between thyroid autoimmunity and fibromyalgic disease severity. Clin Rheumatol. 2007;26(12):2115-2120.

22. Bazzichi L, Rossi A, Zirafa C, et al. Thyroid autoimmunity may represent a predisposition for the development of fibromyalgia? Rheumatol. 2012;32(2):335-341.

23. Suk JH, Lee JH, Kim JM. Association between thyroid autoimmunity and fibromyalgia. Exp Clin Endocrinol Diabetes. 2012;120(7):401-404.

24. Ribeiro LS, Proietti FA. Interrelations between fibromyalgia, thyroid autoantibodies, and depression. J Rheumatol. 2004;31(10): 2036-2040.

25. Cordero MD, Cotán D, del-Pozo-Martín Y, et al. Oral coenzyme Q10 supplementation improves clinical symptoms and recovers pathologic alterations in blood mononuclear cells in a fibromyalgia patient. Nutrition. 2012;28(11-12):1200-1203.

26. Cordero MD, Cano-García FJ, Alcocer-Gómez E, De Miguel M, Sánchez-Alcázar JA. Oxidative stress correlates with headache symptoms in fibromyalgia: coenzyme $\mathrm{Q}_{10}$ effect on clinical improvement. PLoS One. 2012;7(4):e35677.

27. Miyamae T, Seki M, Naga T, et al. Increased oxidative stress and coenzyme Q10 deficiency in juvenile fibromyalgia: amelioration of hypercholesterolemia and fatigue by ubiquinol-10 supplementation. Redox Rep. 2013;18(1):12-19.

28. Meeus M, Nijs J, Hermans L, Goubert D, Calders P. The role of mitochondrial dysfunctions due to oxidative and nitrosative stress in the chronic pain or chronic fatigue syndromes and fibromyalgia patients: peripheral and central mechanisms as therapeutic targets? Expert Opin Ther Targets. 2013;17(9):1081-1089.

29. Castro-Marrero J, Cordero MD, Sáez-Francas N, et al. Could mitochondrial dysfunction be a differentiating marker between chronic fatigue syndrome and fibromyalgia? Antioxid Redox Signal. 2013;19(15): 1855-1860.

30. Neyal M, Yimenicioglu F, Aydeniz A, et al. Plasma nitrite levels, total antioxidant status, total oxidant status, and oxidative stress index in patients with tension-type headache and fibromyalgia. Clin Neurol Neurosurg. 2013;115(6):736-740.

31. Bote ME, García JJ, Hinchado MD, Ortega E. Inflammatory/ stress feedback dysregulation in women with fibromyalgia. Neuroimmunomodulation. 2012;19(6):343-351.

32. Giacomelli C, Bazzichi L, Giusti L, et al. [MALDI-TOF and SELDITOF analysis: "tandem" techniques to identify potential biomarker in fibromyalgia]. Reumatismo. 2011;63(3):165-170. Italian.

33. Altindag O, Gur A, Calgan N, Soran N, Celik H, Selek S. Paraoxonase and arylesterase activities in fibromyalgia. Redox Rep. 2007;12(3): 134-138.

34. Uçeyler N, Häuser W, Sommer C. Systematic review with metaanalysis: cytokines in fibromyalgia syndrome. BMC Musculoskelet Disord. 2011;12:245.

35. Bote ME, Garcia JJ, Hinchado MD, Ortega E. Fibromyalgia: antiinflammatory and stress responses after acute moderate exercise. PLoS One. 2013;8(9):e74524.

36. Pernambuco AP, Schetino LP, Alvim CC, et al. Increased levels of IL-17A in patients with fibromyalgia. Clin Exp Rheumatol. 2013; 31(6 Suppl 79):S60-S63.

37. Ross RL, Jones KD, Bennett RM, Ward RL, Druker BJ, Wood LJ. Preliminary evidence of increased pain and elevated cytokines in fibromyalgia patients with defective growth hormone response to exercise. Open Immunol J. 2010;3:9-18.

38. Iannuccelli C, Di Franco M, Alessandri C, et al. Pathophysiology of fibromyalgia: a comparison with the tension-type headache, a localized pain syndrome. Ann NY Acad Sci. 2010;1193:78-83. 
39. Togo F, Natelson BH, Adler GK, et al. Plasma cytokine fluctuations over time in healthy controls and patients with fibromyalgia. Exp Biol Med (Maywood). 2009;234(2):232-240.

40. Bazzichi L, Rossi A, Massimetti G, et al. Cytokine patterns in fibromyalgia and their correlation with clinical manifestations. Clin Exp Rheumatol. 2007;25(2):225-230.

41. Werle E, Jäkel HP, Müller A, Fischer H, Fiehn W, Eich W. Serum hyaluronic acid levels are elevated in arthritis patients, but normal and not associated with clinical data in patients with fibromyalgia syndrome. Clin Lab. 2005;51(1-2):11-19.

42. Wigley RD, Page B, Chambers EM. Hyaluronic acid serum levels in fibromyalgia, nonspecific arm disorder, and controls. J Rheumatol. 2001;28(11):2563

43. Maes M, Verkerk R, Delmeire L, Van Gastel A, van Hunsel F, Scharpé S. Serotonergic markers and lowered plasma branched-chain-amino acid concentrations in fibromyalgia. Psychiatry Res. 2000;97(1):11-20.

44. Bazzichi L, Palego L, Giannaccini G, et al. Altered amino acid homeostasis in subjects affected by fibromyalgia. Clin Biochem. 2009;42(10-11):1064-1070.

45. Jaschko G, Hepp U, Berkhoff M, et al. Serum serotonin levels are not useful in diagnosing fibromyalgia. Ann Rheum Dis. 2007;66(9): 1267-1268.

46. Offenbaecher M, Bondy B, de Jonge S, et al. Possible association of fibromyalgia with a polymorphism in the serotonin transporter gene regulatory region. Arthritis Rheum. 1999;42(11):2482-2488.

47. Bazzichi L, Giannaccini G, Betti L, et al. Alteration of serotonin transporter density and activity in fibromyalgia. Arthritis Res Ther. 2006;8(4):R99.
48. Dorshkind K, Horseman ND. The roles of prolactin, growth hormone, insulin-like growth factor-I, and thyroid hormones in lymphocyte development and function: insights from genetic models of hormone and hormone receptor deficiency. Endocr Rev. 2000;21(3): 292-312.

49. Ablin JN, Aronov N, Shimon I, et al. Evaluation of leptin levels among fibromyalgia patients before and after three months of treatment, in comparison with healthy controls. Pain Res Manag. 2012;17(2): 89-92.

50. Giusti L, Baldini C, Ciregia F, et al. Is GRP78/BiP a potential salivary biomarker in patients with rheumatoid arthritis? Proteomics Clin Appl. 2010;4(3):315-324

51. Baldini C, Giusti L, Ciregia F, et al. Proteomic analysis of saliva: a unique tool to distinguish primary Sjögren's syndrome from secondary Sjögren's syndrome and other sicca syndromes. Arthritis Res Ther. 2011;13(6):R194.

52. Baldini C, Giusti L, Bazzichi L, et al. Association of psoriasin (S100A7) with clinical manifestations of systemic sclerosis: is its presence in whole saliva a potential predictor of pulmonary involvement? J Rheumatol. 2008;35(9):1820-1824.

53. Bazzichi L, Ciregia F, Giusti L, et al. Detection of potential markers of primary fibromyalgia syndrome in human saliva. Proteomics Clin Appl. 2009;3(11):1296-1304.

54. Samland AK, Sprenger GA. Transaldolase: from biochemistry to human disease. Int J Biochem Cell Biol. 2009;41(7):1482-1494.
Current Biomarker Findings

\section{Publish your work in this journal}

Current Biomarker Findings is an international, peer-reviewed, open access journal publishing original research, reports, reviews and commentaries on all areas of biomarker research. The manuscript management system is completely online and includes a very quick and fair

\section{Dovepress}

peer-review system. Visit http://www.dovepress.com/testimonials.php to read real quotes from published authors. 